\title{
DIURNAL VARIATION \\ OF THE MELANIN-CONCENTRATING HORMONE LEVEL IN THE HYPOTHALAMUS
}

\author{
Balázs Gerics, Ferenc Szalay, Péter Sótonyi and Veronika JancsiK* \\ Department of Anatomy and Histology, University of Veterinary Medicine, \\ H-1078 Budapest, István utca 2, Hungary
}

(Received: September 8, 2016; accepted: October 17, 2016)

\begin{abstract}
Melanin-concentrating hormone ( $\mathrm{MCH})$, the neuropeptide produced mainly in the hypothalamus, plays an operative role in regulating food intake and the sleep/wake cycle. Considering that these physiological functions pursue diurnal variations, we checked whether the total hypothalamic MCH level depends on the time of the day. The aggregated $\mathrm{MCH}$ peptide content of the whole $\mathrm{MCH}$ neuron population was significantly higher at the end of the sleeping period (lights on), than at the end of the active period (lights off). This result, together with earlier observations, indicates that in contrast to the $\mathrm{MCH}$ gene expression, the level of $\mathrm{MCH}$ peptide is object of circadian variation in the hypothalamus.
\end{abstract}

Keywords: Neuropeptide - circadian rhythm - energy homeostasis - sleep

\section{INTRODUCTION}

The hypothalamus holds the light-driven, genetically defined biological clock of mammals, as well as key structures necessary for the integration of environmental factors, such as food availability, social and meteorological conditions $[8,19]$.

It is particularly important to handle energy homeostasis during different levels of food availability. A complex hypothalamic neural network, including melanin-concentrating hormone $(\mathrm{MCH})$ and orexin producing neurons, two discrete but spatially overlapping neuron populations contribute to the regulation of energy homeostasis on a large scale. Interestingly, the very same neurons are partakers of the circuit which influence the sleep-wake cycle.

In the mammalian brain $\mathrm{MCH}$ producing neurons occur within a rostro-caudally restricted region of the hypothalamus, first of all in the lateral hypothalamic area (LHA) and in the zona incerta (ZI) [3, 17]. Mapping of the distribution of MCH neuron perikarya and their projections in the rat $[1,2,5]$ and mouse brain $[3,4]$ revealed the presence of $\mathrm{MCH}$ neuron somata in the lateral hypothalamic area, including the perifornical region, the rostromedial zona incerta, the posterior hypothalamic nucleus, and the capsule of the ventromedial nucleus, as often as not breaching the cyto-

*Corresponding author; e-mail address: jancsik.veronika@univet.hu 
architectonic borders. Notwithstanding this restricted localization of the perikarya, $\mathrm{MCH}$ conveying projections reach a wide variety of target areas within the CNS and on the periphery. Regions affected include, among others, those involved in regulating feeding behaviour (e.g. lateral septum, nucleus accumbens) and those promoting arousal state (e.g. tuberomammillary nucleus, basal forebrain, locus coeruleus, ventrolateral periaqueductal gray, dorsal raphe), making this neuropeptide a potential candidate to constitute functional link between physiological processes [16, 22].

Two MCH neuron subpopulations are present in the hypothalamus: neurons containing $\mathrm{MCH}$ as the only neuropeptide - classified as type A MCH neurons - and those expressing another neuropeptide, the cocaine- and amphetamine regulated transcript (CART) and the neurokinin 3 receptor as well, denoted as type B MCH neurons. The two subpopulations are partially segregated within the hypothalamus: laterally and caudally the majority of the $\mathrm{MCH}$ neurons belong to type A, while type $\mathrm{B} \mathrm{MCH}$ neurons are predominating medially and in the rostral ZI. Due the imperfectness of their spatial segregation, immunohistochemical identification is needed to tell the perikarya of the two subpopulations apart. The projections of type A and type B $\mathrm{MCH}$ neurons are principally directed towards the brainstem and the telencephalon, respectively $[4,5]$. The functional importance of the coexpression of $\mathrm{MCH}$ and CART is unknown for the time being.

$\mathrm{MCH}$ has for long been known as a potent orexigenic neuropeptide, influencing both feeding behaviour and energy expenditure $[13,14,16]$. Fasting is known to increase the expression of $\mathrm{MCH}$ mRNA [18]. $\mathrm{MCH}$ is also a central player in the promotion and stabilization of sleep [7, 10-12, 21]. It has been suggested that $\mathrm{MCH}$ is affecting the pathophysiology of depression as well [21].

Considering the circadian rhythm of these physiological processes, it is plausible to suppose diurnal variations in the functioning of the MCHergic system. Nevertheless, available data are scanty.

Harthoorn et al. [9] analysed individual $\mathrm{MCH}$ neurons in the rat hypothalamus. They detected constant levels of MCH mRNA and peptide content at ZT2 and ZT14. (ZT stands for Zeitgeber time - "time giver" in German language. ZT0 traditionally corresponds to turning the lights on.) Similarly, statistically significant correlations were not found between the periodic expression of $\mathrm{MCH}$ and orexin genes with food intake in mouse hypothalamus [20].

In a recent comprehensive study, Dias Abdo Agamme et al. [6] measured the level of the MCH peptide by ELISA in the cerebrospinal fluid, along with the expression of the $\mathrm{MCH}$ gene (Pmch) in the hypothalamus, and the expression of the $\mathrm{MCH}$ receptor gene in the frontal cortex and the hippocampus, during active and sleep phases. Under undisturbed conditions all parameters were independent from the phases, excepting the $\mathrm{MCH}$ receptor expression, which was significantly higher when sleep predominated. Disturbances of the sleep-wake cycle, on the other hand, induced day/ night differences in CSF levels of MCH and in Pmch gene expression.

In the present study, we have undertaken to test directly the $\mathrm{MCH}$ levels in the mouse hypothalamus at the end of the active and sleep phases by immunohistochemical assessment of the total $\mathrm{MCH}$ peptide content. 


\section{MATERIALS AND METHODS}

\section{Animals and tissue preparation}

Young adult (30-34 g) Crl:NMRI BR male mice (Toxi-Coop Zrt, Hungary) were used during the experiments.

Mice were kept under standard conditions, with water and rodent chow ad libitum. A 12/12 h dark/light cycle was applied (lights on at 8:00 AM and off at 8:00 PM). Animals were divided into two groups: ZT0 and ZT12 group member mice were exterminated immediately after the end of the dark period, and immediately after the end of the light period, respectively.

Transcardial perfusion was done under anaesthesia with glutaraldehyde supplemented Zamboni's fixative (4\% paraformaldehyde, $0.4 \%$ glutaraldehyde, $15 \%$ saturated picric acid in $0.1 \mathrm{M}$ phosphate buffer, $\mathrm{pH}$ 7.4). After removal from the skull, brains were further incubated overnight at $+5{ }^{\circ} \mathrm{C}$ in the same fixative without glutaraldehyde. A $50 \mu \mathrm{m}$ coronal sections were cut with a vibratome.

The study was conducted in accordance with the 2010/63/EU Directive, with written permission of the institutional Animal Health and Welfare Committee.

\section{MATERIALS}

\section{Primary antibody}

Anti-MCH: polyclonal, raised in rabbit (Sigma-Aldrich, M8440). Immunogen: keyhole limpet hemocyanin (KLH)-coupled synthetic peptide corresponding to human $\mathrm{MCH}$.

\section{Techniques}

\section{Detection of MCH immunoreactivity}

Sections containing the hypothalamus were processed for immunohistochemistry free-floating. All incubations and washings were carried out at room temperature $\left(20-23^{\circ} \mathrm{C}\right)$.

Blocking of the non-specific binding sites with $20 \%$ normal donkey serum (Life Technologies) was followed by overnight incubation with anti-MCH (Sigma-Aldrich, dilution: $\times 10,000$ ). Biotinylated anti-rabbit $\operatorname{IgG}$ secondary antibody (Amersham, dilution: $\times 100$ ) and avidin-biotin-horseradish peroxidase (VECTASTAIN Elite ABC HRP Kit, Vector laboratories, Cat. No: PK-6100) were applied according to the manufacturer's instructions. The 3,3'diaminobenzidine (DAB) was used as chromogen. To assure the comparability of the results, the immunodetection procedure for all mice (ZT0 and ZT12 collectively) has been performed in parallel, as a single series. Complete rostro-caudal series of MCH neuron containing sections were considered 
for further densitometric and statistical evaluation. Incomplete series (with failing or damaged sections) have been disregarded. At the end, three series of sections from each ZT group have been evaluated.

\section{Densitometric evaluation}

Immunolabelled sections were monitored and photographed by a Leica DM750 microscope equipped with a Leica DMC2900 camera. On each section left and right sides of the hypothalamus were separately photographed with standardized illumination by using $\times 10$ magnification. Photos $(2048 \times 1536$ pixels $)$ were converted into 8 bit grayscale for the purpose of the densitometric evaluation, done with the Scion Image Programme, version v4.02 $\beta$ for Windows. Thresholding to the optical density level of the background staining was performed. A constant sized reference frame has been positioned onto each photo to include all immunopositive neurons. The resulting data (positivity in arbitrary units) reflect the immunopositive area within the reference frame (Fig. 1). The number of data varied between 34 and 42 per brain. These data were used as counts in the statistical analysis.

\section{Statistical analysis}

Statistical analysis was done by using the Stata13 software (StataCorp LP, USA). Unpaired $t$-tests have been performed at $95 \%$ confidence level. To check incidental consequences of slightly oblique section planes, density counts of left and right sides were compared. No significant differences have been found. Aggregate density counts at ZT0 and ZT12 (122 and 121) counts, respectively, as well as the means of density counts of the individual animals within and between the groups, have also been evaluated by $t$-test.

\section{RESULTS}

Density count data, proportional with the level of immunopositivity have been obtained by densitometric evaluation of the immunolabelled brain sections, as illustrated in Figure 1. Density count data appear on an arbitrary scale. Comparability of the data has been guaranteed by careful standardization of all experimental conditions. Total hypothalamic $\mathrm{MCH}$ levels of individual mice have been assessed by the addition of density count data in all coronal hypothalamic sections depicting $\mathrm{MCH}$ neurons. Statistical comparison of aggregate density counts of all sections obtained from ZT0 and ZT12 mice has been performed. Box and whiskers plots (Fig. 2) indicate that the distribution of density readings is highly skewed. Even if the data do not follow normal distribution, due to the large number of data (121 and 122, respectively), the $t$-test (with equal variances) could be used for statistical comparison. 


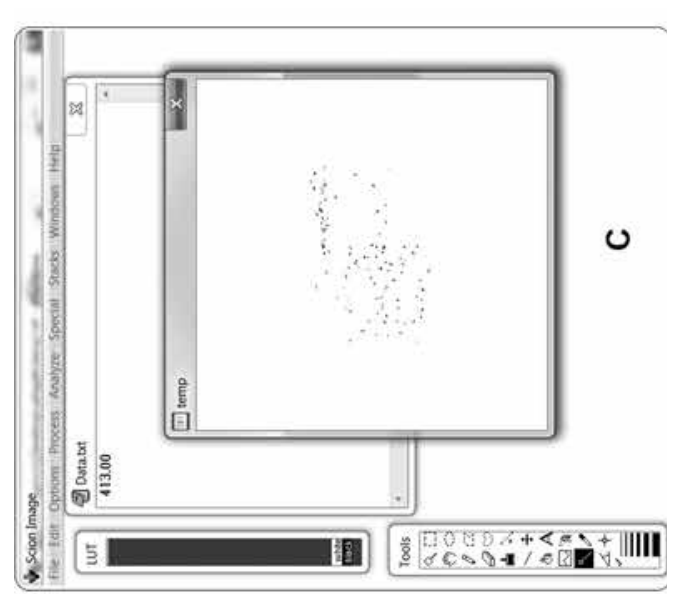

灵官它嵒官 可 罢电品 宅可 $\exists$ 웡

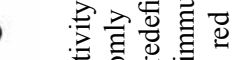
记 这

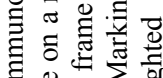

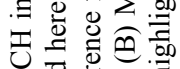
$\sum$ 语

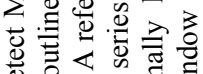

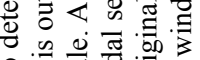

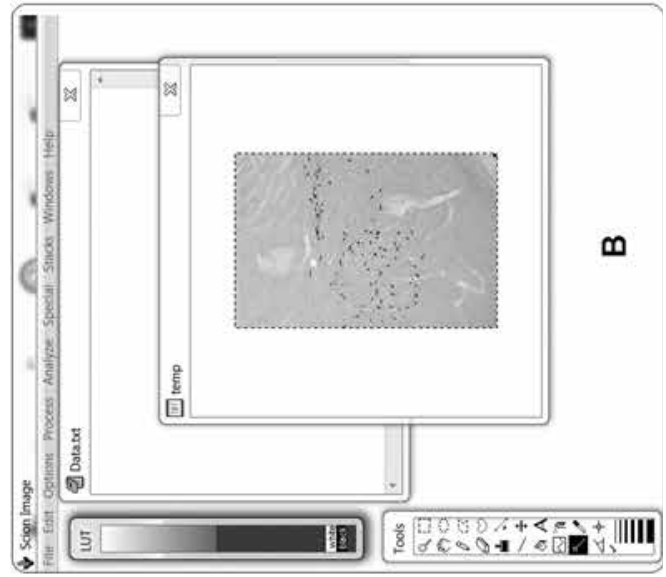
论

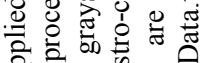

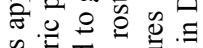

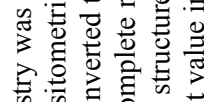

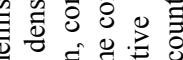
응.

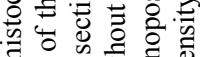

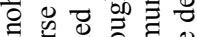

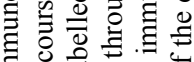
E용

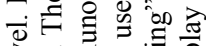

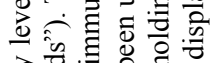

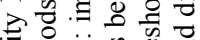
造要

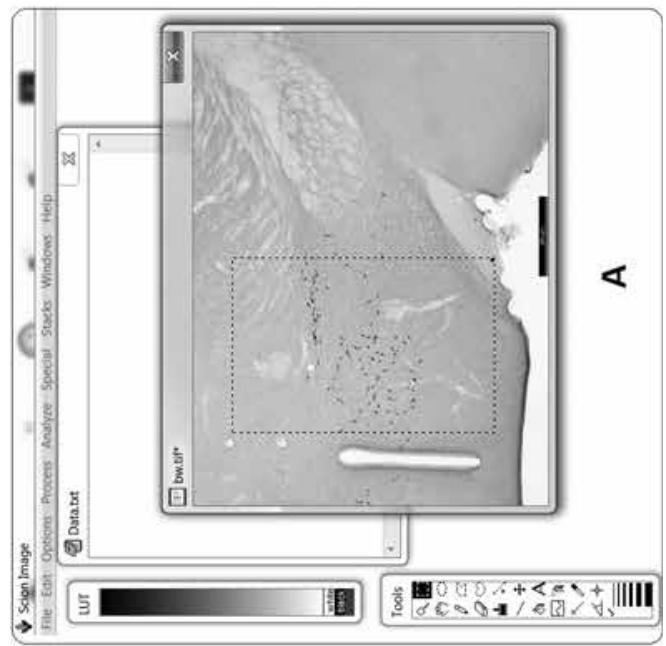

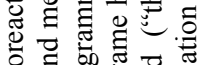
为 音

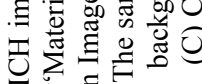

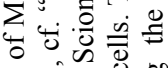

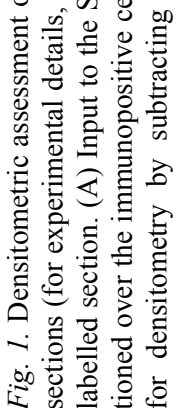




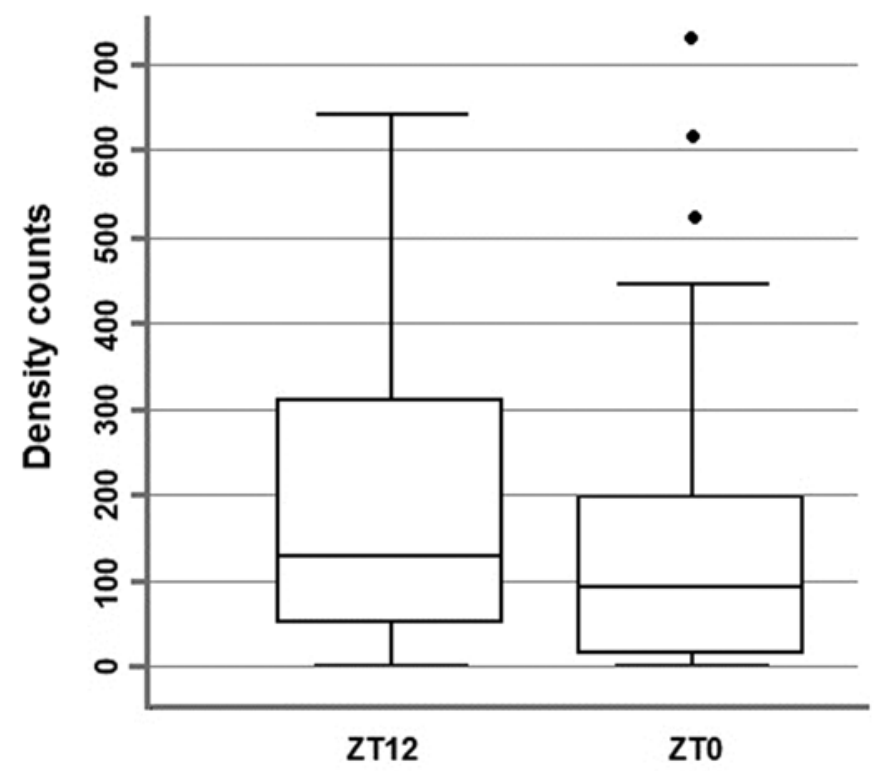

Fig. 2. Box and whisker plots of density count data demonstrate significantly lower $\mathrm{MCH}$ level in the hypothalamus at the end of the active period (ZT0) than at the end of the sleeping period (ZT12)

The means, CI (95\%) and medians for ZT0 and ZT12 were 122.57 (98.70-146.4), 87 and 182.04 (153.6-210.5) and 128, respectively. The difference between the data at the two diurnal time points, ZT0 and ZT12 was significant ( $\mathrm{p}=0.0017$, CI $(95 \%)$ : 22.54-96.40). Non-parametric tests (Kolmogorov-Smirnov and Shapiro-Wilk) also showed significant difference between ZT0 and ZT12 (data not shown).

Although the observed difference is highly significant, extension of the experiments to a higher number of animals is planned.

\section{DISCUSSION}

$\mathrm{MCH}$ neurons are known to be involved in circuits influencing feeding behaviour and sleep-wake rhythm. Therefore, it seemed to be highly conceivable that their peptide content followed circadian pattern.

Former studies addressed this point by diverse approaches. Harthoorn et al. [9] selected $25 \mathrm{MCH}$ neurons in the rat hypothalamus and found that their $\mathrm{MCH}$ content did not depend on the diurnal time point. Likewise, the expression profile of the Pmch gene did not exhibit particularly strong circadian expression profile in mice [20], similarly to several other genes regulating food intake. Dias Abdo Agamme et al. [6] also observed absence of day/night differences in Pmch expression in the rat hypothalamus under undisturbed conditions. 
It is worth noting, however, that the time of the day has been found to influence the response of the MCHergic system to abnormal conditions, e.g. fasting [19] or sleep disruption [6].

The aim of the work presented here was to compare the amount of $\mathrm{MCH}$ peptide present in the whole $\mathrm{MCH}$ neuron population of mice at the end of the active (dark) and the sleeping (light) periods. $\mathrm{MCH}$ peptide levels were assessed by densitometric evaluation of immunohistochemically labelled sections. MCH content of the whole hypothalamic $\mathrm{MCH}$ neuron population was calculated by adding up the $\mathrm{MCH}$ content of all coronal sections containing $\mathrm{MCH}$ neuron perikarya. Type A and type $\mathrm{B} \mathrm{MCH}$ neuron subpopulations were not distinguished. The $\mathrm{MCH}$ level calculated this way has proven to be significantly lower at the end of the active period (ZT0) as compared to the end of the sleeping period (ZT12). This result implies that the replenishment and/or the depletion of the MCH pool depends on the time of the day. Depletion of the hypothalamic $\mathrm{MCH}$ pool is supposed to depend mostly on the activity dependent transport of the neuropeptide to its multiple target sites. For the time being, the rate and regulation of this transport process is unidentified. To address this issue, the possible diurnal variation of the $\mathrm{MCH}$ level in the various target areas should be studied as well. As far as the replenishment of the pool is concerned, earlier results indicated that the level of Pmch gene expression, as characterized by the MCH mRNA level, does not depend on the time of the day $[6,20]$. It is to be considered, that beyond transcription, formation of mature $\mathrm{MCH}$ involves translation of the mRNA to preproMCH and the posttranslational proteolytic cleavage of the emerging prohormone $[15,23]$ as well. The possible circadian rhythm of these processes was not addressed yet.

As a conclusion, the observed diurnal variation of the $\mathrm{MCH}$ peptide level in the hypothalamus is verisimilarly of physiological importance. Further investigations are needed to ascertain the underlying mechanisms.

\section{ACKNOWLEDGEMENTS}

The authors thank Mihály Krámer for his help in statistical analysis. The expert technical assistance of Tünde Magyar and the participation of Gregor Merz in the experiments are highly appreciated. This work has been supported by the Hungarian Research Fund, OTKA/National Research, Development and Innovation Office, NKFIH (K 81419). This publication was supported by the 11475-4/2016/FEKUT Grant of the Hungarian Ministry of Human Resources.

\section{REFERENCES}

1. Bittencourt, J. C., Presse, F., Arias, C., Peto, C., Vaughan, J., Nahon, J. L., Vale, W., Sawchenko, P. E. (1992) The melanin-concentrating hormone system of the rat brain: an immuno- and hybridization histochemical characterization. J. Comp. Neurol. 319, 218-245.

2. Bittencourt, J. C. (2011) Anatomical organization of the melanin-concentrating hormone peptide family in the mammalian brain. Gen. Comp. Endocrinol. 172, 185-197.

3. Croizier, S., Cardot, J., Brischoux, F., Fellmann, D., Griffond, B., Risold, P. Y. (2013) The vertebrate diencephalic MCH system: a versatile neuronal population in an evolving brain. Front Neuroendocrinol. $34,65-87$. 
4. Croizier, S., Franchi-Bernard, G., Colard, C., Poncet, F., La Roche, A., Risold, P. Y. (2010) A comparative analysis shows morphofunctional differences between the rat and mouse melanin-concentrating hormone systems. PLoS One 5:e15471.

5. Cvetkovic, V., Brischoux, F., Jacquemard, C., Fellmann, D., Griffond, B., Risold, P. Y. (2004) Characterization of subpopulations of neurons producing melanin-concentrating hormone in the rat ventral diencephalon. J. Neurochem. 91, 911-919.

6. Dias Abdo Agamme, A. L., Aguilar Calegare, B. F., Fernandes, L., Costa, A., Lagos, P., Torterolo, P., D'Almeida, V. (2015) MCH levels in the CSF, brain preproMCH and MCHR1 gene expression during paradoxical sleep deprivation, sleep rebound and chronic sleep restriction. Peptides 74, 9-15.

7. Fraigne, J. J., Peever, J. H. (2013) Melanin-concentrating hormone neurons promote and stabilize sleep. Sleep 36, 1767-1768.

8. Gooley, J. J., Saper, C. B. (2010) Anatomy of the mammalian circadian system. In: Kryger, M. H., Reth, T., Dement, W. C. (eds), Principles and Practice of Sleep Medicine (5th ed.). Elsevier Health Sciences.

9. Harthoorn, L. F., Sañé, A., Nethe, M., Van Heerikhuize, J. J. (2005) Multi-transcriptional profiling of melanin-concentrating hormone and orexin-containing neurons. Cell. Mol. Neurobiol. 25, 1209-1223.

10. Hassani, O. K., Lee, M. G., Jones, B. E. (2009) Melanin-concentrating hormone neurons discharge in a reciprocal manner to orexin neurons across the sleep-wake cycle. Proc. Natl Acad. Sci. USA 106, 2418-2422.

11. Jego, S., Glasgow, S. D., Herrera, C. G., Ekstrand, M., Reed, S. J., Boyce, R., Friedman, J., Burdakov, D., Adamantidis, A. R. (2013) Optogenetic identification of a rapid eye movement sleep modulatory circuit in the hypothalamus. Nat. Neurosci. 16, 1637-1643.

12. Konadhode, R. R., Pelluru, D., Blanco-Centurion, C., Zayachkivsky, A., Liu, M., Uhde, T., Glen, W. B. Jr., van den Pol, A. N., Mulholland, P. J., Shiromani, P. J. (2013) Optogenetic stimulation of MCH neurons increases sleep. J. Neurosci. 33, 10257-10263.

13. Macneil, D. J. (2013) The role of melanin-concentrating hormone and its receptors in energy homeostasis. Front. Endocrinol. (Lausanne) 4:49. doi: 10.3389/fendo.2013.00049. eCollection 2013

14. Nahon, J. L. (2006) The melanocortins and melanin-concentrating hormone in the central regulation of feeding behavior and energy homeostasis. C. R. Biol. 329, 623-638.

15. Nahon, J. L., Presse, F., Bittencourt, J. C., Sawchenko, P. E., Vale, W. (1989) The rat melanin-concentrating hormone messenger ribonucleic acid encodes multiple putative neuropeptides coexpressed in the dorsolateral hypothalamus. Endocrinology 125, 2056-2065.

16. Pissios, P., Bradley, R. L., Maratos-Flier, E. (2006) Expanding the scales: The multiple roles of MCH in regulating energy balance and other biological functions. Endocr. Rev. 27, 606-620.

17. Pissios, P., Maratos-Flier, E. (2003) Melanin-concentrating hormone: from fish skin to skinny mammals. Trends Endocrinol. Metab. 14, 243-248.

18. Qu, D., Ludwig, D. S., Gammeltoft, S., Piper, M., Pelleymounter, M. A., Cullen, M. J., Mathes, W. F., Przypek, R., Kanarek, R., Maratos-Flier, E. (1996) A role for melanin-concentrating hormone in the central regulation of feeding behaviour. Nature 380, 243-247.

19. Saper, C. B., Fuller, P. M. (2007) Inducible clocks: living in an unpredictable world. Cold Spring Harb. Symp. Quant. Biol. 72, 543-550.

20. Stütz, A. M., Staszkiewicz, J., Ptitsyn, A., Argyropoulos, G. (2007) Circadian expression of genes regulating food intake. Obesity (Silver Spring) 15, 607-615.

21. Torterolo, P., Scorza, C., Lagos, P., Urbanavicius, J., Benedetto, L., Pascovich, C., López-Hill, X., Chase, M. H., Monti, J. M. (2015) Melanin-concentrating hormone (MCH): Role in REM sleep and depression. Front Neurosci. 9:475. doi: 10.3389/fnins.2015.0047

22. Urstadt, K. R., Stanley, B. G. (2015) Direct hypothalamic and indirect trans-pallidal, trans-thalamic, or trans-septal control of accumbens signaling and their roles in food intake. Front Syst. Neurosci. 9:8. doi: 10.3389/fnsys.2015.00008.

23. Viale, A., Ortola, C., Hervieu, G., Furuta, M., Barbero, P., Steiner, D. F., Seidah, N. G., Nahon, J. L. (1999) Cellular localization and role of prohormone convertases in the processing of pro-melaninconcentrating hormone in mammals. J. Biol. Chem. 274, 6536-6545. 